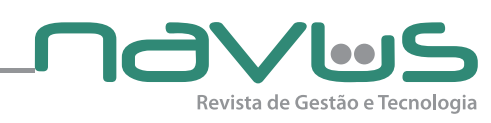

\title{
Estratégias de inovação e crescimento sustentadas por práticas da gestão do conhecimento
}

\author{
Leonardo Leocádio Coelho de Souza' \\ Rayanne Ribeiro de Holanda ${ }^{2}$ \\ Sheilane Pereira de Sousa ${ }^{3}$ \\ Evandro Cardoso da Costa ${ }^{4}$
}

\begin{abstract}
RESUMO
Para formular ou alterar seu comportamento no intuito de adquirir maior competitividade no mercado, uma empresa precisa primeiramente trabalhar uma série de requisitos. Entre eles, destacam-se a definição dos objetivos e metas da empresa, a escolha da melhor estratégia a ser implementada e a verificação da disponibilidade dos recursos necessários. Alinhada a essa realidade, uma visão bastante difundida na literatura é a ideia de que os gestores das Pequenas e Médias Empresas (PMEs) necessitam olhar além dos produtos da empresa e focar na identificação de fontes de conhecimento especializado que possam atender às demandas e alavancar vantagens competitivas com base na melhoria do desempenho dos seus processos. Diante disso, este artigo tem como objetivo desenvolver uma metodologia de diagnóstico e avaliação da capacidade de crescimento e inovação das PMEs que, de maneira complementar, combine a metodologia Aprender a Crescer com as práticas da gestão do conhecimento, levando em consideração as necessidades do pequeno empresário brasileiro. Trata-se de um estudo de cunho exploratório e descritivo, com abordagem qualitativa, que possibilita a compreensão de quais práticas da gestão do conhecimento, combinadas com a metodologia Aprender a Crescer, potencializam a capacidade de inovação e crescimento das PMEs. Portanto, como principal contribuição deste trabalho está a possibilidade de avaliar o desempenho e a relevância das práticas relacionadas à capacidade de reconhecer oportunidades para o crescimento, desenvolver e comunicar as estratégias e os objetivos, realizar as oportunidades e gerenciar o crescimento.
\end{abstract}

Palavras-chave: Práticas da gestão do conhecimento. Inovação. Pequenas e médias empresas. Vantagem competitiva. Aprender a crescer.

\section{INTRODUÇÃO}

As pequenas e médias empresas (PMEs) têm uma expressiva importância na economia mundial, representando uma significativa parcela do Produto Interno Bruto (PIB) nos países desenvolvidos e nos

\footnotetext{
${ }^{1}$ Doutor em Engenharia e Gestão do Conhecimento. Universidade Federal do Maranhão - leoleocadio@gmail.com

${ }^{2}$ Aluna de Graduação em Direito. Universidade Federal do Maranhão - mrayannedeholanda@gmail.com

${ }^{3}$ Aluna de Graduação em Ciências Contábeis. Universidade Federal do Maranhão - sheilaneps@gmail.com

${ }^{4}$ Aluno de Graduação em Ciências Contábeis. Universidade Federal do Maranhão - evando.cardoso@gmail.com
} 
países em desenvolvimento (SANTOS; VARVAKIS, 1999). Contudo, o ambiente econômico turbulento no qual as PMEs estão inseridas dificulta o seu crescimento sustentado e a sua sobrevivência ao longo do tempo.

Desse modo, a capacidade de inovação é importante para as empresas que querem crescer e se manter sustentáveis no mercado. Alguns dos fatores limitadores para a inovação dessas empresas são aqueles ligados aos fluxos de informação e conhecimento, tais como escassez de capital, ausência de qualificação em gestão e dificuldades em obter informações técnicas e know-how para inovação (MULLER; ZENKER, 2001).

Diante desses obstáculos, a metodologia Aprender a Crescer (HARDWIG; BERGSTERMANN; NORTH, 2011) surge como uma proposta de instrumento estratégico, gerencial e operacional, que busca a eficácia empresarial da pequena e média empresa, possibilitando-a vir a se tornar uma empresa dinâmica. 0 enfoque dessa metodologia permite melhorar as capacidades inovadoras da empresa, assim como fidelizar e desenvolver as capacidades dos colaboradores.

Ainda, considerando a turbulência do entorno empresarial enfrentado pelas PMEs, a literatura especializada também reconhece a identificação, gestão e uso dos recursos de conhecimento dessas empresas, sob a lente da Gestão do Conhecimento e mediante suas práticas, pelas quais se privilegia o conhecimento como elemento alavancador de vantagem competitiva (PARRILLI; ARANGUREN; LARREA, 2010; WANG; WANG; HORNG, 2010).

Diante disso, este trabalho surge como uma proposta de estudo sobre as estratégias de crescimento e inovação das PMEs, a partir das práticas da gestão do conhecimento e da metodologia Aprender a Crescer, com o intuito de contribuir com trabalhos anteriores (HARDWIG; BERGSTERMANN; NORTH, 2011; SOUZA, 2011; MENEZES et al, 2012; NORTH; SILVA NETO; CALLE, 2013).

Para tanto, a estrutura deste artigo apresenta-se dividida em cinco partes. Além desta introdução, o item 2 expõe o entendimento da literatura especializada sobre a metodologia Aprender a Crescer, as práticas da gestão do conhecimento e o contexto das PMEs. No item 3, são apresentados os procedimentos metodológicos adotados para a construção da metodologia de diagnóstico e a avaliação proposta por este trabalho. 0 item 4 relata a análise dos dados referentes a essa metodologia; o item 5 traz algumas considerações acerca deste artigo e, por fim, são apresentadas as referências utilizadas para embasar esta pesquisa.

\subsection{Objetivo geral}

De acordo com o exposto, o objetivo deste trabalho consiste no desenvolvimento de uma metodologia de diagnóstico e avaliação da capacidade de crescimento e inovação das PMEs, que combine a metodologia Aprender a Crescer com as práticas da gestão do conhecimento levando em consideração as necessidades do pequeno empresário no Brasil.

\subsection{Objetivos específicos}

Para o alcance do objetivo geral, estabeleceram-se os seguintes objetivos específicos: (i) identificar as práticas da gestão do conhecimento na literatura especializada; (ii) selecionar um conjunto de práticas da gestão do conhecimento que potencializam a inovação e crescimento das PMEs; e (iii) desenvolver uma metodologia de diagnóstico que combine práticas da gestão do conhecimento com a metodologia Aprender a Crescer.

\section{FUNDAMENTAÇÃO TEÓRICA}

Para sustentar a proposta deste estudo, faz-se necessário direcionar as discussões teóricas sobre a metodologia Aprender a Crescer - discutida por Hardwig, Bergstermann e North (2011) -, as práticas da gestão do conhecimento e o contexto das PMEs.

\subsection{Metodologia Aprender a Crescer}

No cenário atual, caracterizado por turbulências, dinamismo e alta complexidade, as PMEs precisam ser capazes de manter vantagens competitivas. Nesse cenário de mudanças, essas empresas são desafiadas a incorporar novos conhecimentos à base de conhecimento já existente na empresa.

Em virtude das características das PMEs e da influência do contexto no qual estão inseridas, vários estudiosos têm apontado para a necessidade de abordagens teóricas desenvolvidas especificamente para estudar esses tipos de empresas, e, na tentativa de preencher essa lacuna, surge a Metodologia Aprender a 
Crescer, desenvolvida pelos autores Hardwig, Bergstermann e North (2011), e aplicada com sucesso em 124 PMEs inovadoras e em crescimento na Alemanha.

Baseada no conceito de capacidades dinâmicas, tal Metodologia é um instrumento estratégico, gerencial e operacional, que busca a eficácia empresarial da pequena e média empresa, tornando-as empresas dinâmicas (NORTH; SILVA NETO; CALLE, 2013). Para outro grupo de autores (TEECE; PISANO; SHUEN, 1997), capacidade dinâmica pode ser entendida como a habilidade das empresas de integrar, construir e reconfigurar suas competências internas e externas, com o objetivo de enfrentar os desafios constantes do mercado. Assim, em ambientes turbulentos, as PMEs precisam reconhecer as mudanças e reagir com efetividade e rapidez.

Essas capacidades, segundo Teece (2007), são apresentadas em três distintas capacidades: (i) sensing, entendido como a capacidade de sentir, perceber e configurar as oportunidades e ameaças do mercado; (ii) seizing, para aproveitar as capacidades percebidas; e (iii) managing and transforming, como a capacidade de manter a competitividade por meio da melhoria contínua, combinando, protegendo e reconfigurando o negócio da organização.

De acordo com a Metodologia Aprender a Crescer, as competências essenciais para o crescimento são desenvolvidas com a contribuição da Roda do Crescimento (NORTH; SILVA NETO; CALLE, 2013) apresentada na Figura 1. A referida Metodologia foi desenvolvida com base nos conceitos do ciclo de crescimento das empresas e tendo suas questões elaboradas e organizadas em quatro desafios a serem perseguidos pelas PMEs no processo de crescimento: (1) reconhecer oportunidades para o crescimento; (2) desenvolver e comunicar estratégias de crescimento; (3) realizar as oportunidades de crescimento; e (4) gerenciar o crescimento.

Cada desafio possui dois grupos, configurando oito no total, com o objetivo de proporcionar a superação dos quatro desafios propostos, de forma que a metodologia consiste num questionário estruturado com dezesseis perguntas, que serão exploradas em maior detalhe na seção 4 deste trabalho (Apresentação e Análise dos Resultados).

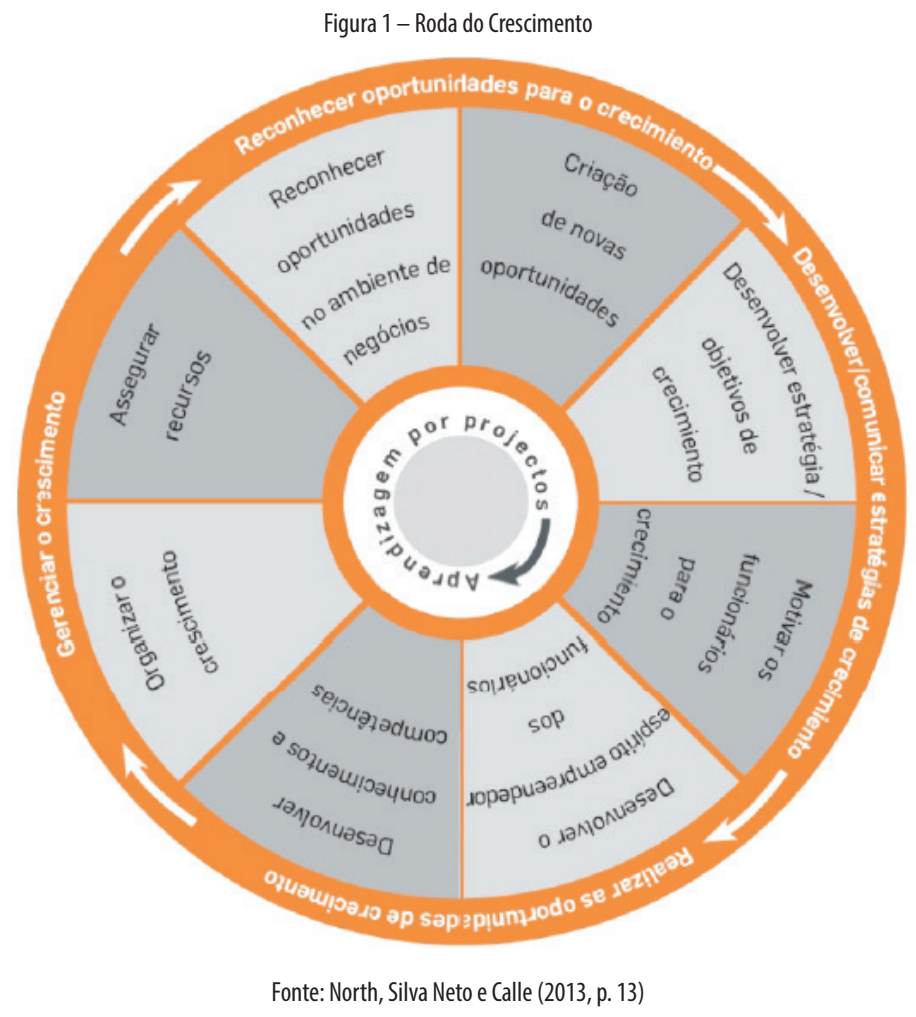

Vale salientar que a Metodologia Aprender a Crescer utiliza um enfoque baseado na aprendizagem, implementando soluções operacionais por intermédio de projetos de aprendizagem. 0 enfoque integrado permite melhorar as capacidades inovadoras da empresa, assim como fidelizar e desenvolver as capacidades dos colaboradores. 
Alinhada à referida metodologia e considerando a turbulência do entorno empresarial enfrentado pela PMEs, paralelamente, a literatura especializada também reconhece a identificação, gestão e uso dos recursos de conhecimento das PMEs sob a lente da Gestão do Conhecimento, na qual se privilegia o conhecimento como elemento alavancador de vantagem competitiva (PARRILLI; ARANGUREN; LARREA, 2010; WANG; WANG; HORNG, 2010).

Contudo, Schreiber et al (2002) destacam que, apesar de o conhecimento ser apontado como fator importante para o sucesso organizacional, apenas $20 \%$ do conhecimento disponível na organização é utilizado.

Essas constatações reforçam a necessidade do desenvolvimento de estudos que auxiliem as PMEs a estabelecerem uma postura que deve ir além das simples análises tradicionais de custo e formação de preços, uma vez que a capacidade de identificar o conhecimento relevante para gerir e extrair valor é apontada como a única vantagem competitiva sustentável para as empresas (DRUCKER, 1994; GRANT, 1996; DAVENPORT; PRUSAK, 1998; LLORIA, 2008; BISHOP et al, 2008).

Desse modo, avaliar a capacidade de inovação e de crescimento das PMEs, pela identificação e/ou implementação das práticas da gestão do conhecimento, configura-se em uma oportunidade para a melhoria dos serviços prestados, além da redução dos riscos de mortalidade inerentes a essas empresas.

\subsection{Práticas de Gestão do Conhecimento}

Segundo North (2010), nos países industriais de ponta só é possível ser competitivo de forma duradoura quando se usa melhor o fator de produção de conhecimento. Desse modo, gerenciar o conhecimento, como um dos fatores de produção mais importantes das organizações, tornou-se imprescindível.

De acordo com os objetivos deste trabalho e tomando como base as definições e perspectivas apresentadas por Skyrme e Amidon (1997), Davenport e Prusak (1998), OECD (2003), APQC (2006) e Lloria (2008), entende-se que a gestão do conhecimento envolve qualquer ação ou prática relacionada aos processos de criação, disseminação e uso do conhecimento para atingir os objetivos da organização.

0 processo de criação de conhecimento pode ser definido como o compartilhamento do conhecimento mental, emocional e ativo, resultando em valor agregado para a organização (POPADIUK; CHO0, 2006). Nesse sentido, é o esforço de identificar, adquirir ou criar novo conhecimento com o objetivo de agregar valor para a organização.

Disseminar o conhecimento é disponibilizá-lo aos membros da empresa, permitindo que sejam usados para benefício dos objetivos da organização. 0 uso de TCIs, por exemplo, aumenta as possibilidades de disseminação do conhecimento, ao torná-lo disponível e de fácil acesso para o uso dos membros da empresa (SOUZA, 2011).

O uso do conhecimento, por sua vez, conforme Kogut e Zander (1992), é a capacidade da organização de explorar seus conhecimentos e, com isso, gerar mais aplicações e melhores resultados. É, portanto, a capacidade de colocar o conhecimento adquirido em ação organizacional.

Segundo Souza (2011), algumas práticas facilitam a condução dos processos de criação, disseminação e uso do conhecimento. Essas práticas são compreendidas por Feldman e Pentland (2003) como rotinas organizacionais referentes a padrões repetitivos de ações interdependentes, realizadas por múltiplos membros organizacionais envolvidos na realização das tarefas e processos.

Diante da importância do conhecimento como o grande diferencial das organizações (DRUCKER, 1994; LLORIA, 2008), conduzir seus processos de criação, disseminação e uso torna-se necessário. Para isso, as práticas da gestão do conhecimento podem viabilizar tal condução.

A gestão eficiente do recurso conhecimento, a combinação de novas capacidades e um olhar atento às oportunidades do mercado é tarefa difícil, e esse tem sido o desafio enfrentado pelos gestores das PMEs brasileiras em mercados de intensa competição. Assim sendo, compreender como as PMEs enfrentam tais desafios, utilizando as práticas da gestão do conhecimento é uma das principais contribuições deste estudo.

\subsection{As pequenas e médias empresas}

O mercado é um ambiente dinâmico que agrega os mais variados tipos de organizações. Nele, estão inseridas as PMEs, que demonstram, por meio de indicadores, sua importância para promoção do crescimento econômico, geração de empregos, renda e melhoria nas condições de vida da população. Este segmento empresarial igualmente tem sido capaz de dinamizar a economia dos municípios e bairros das grandes metrópoles (PORTAL BRASIL, 2010).

Sérgio Risola, diretor executivo do Centro de Inovação, Empreendedorismo e Tecnologia (Cietec), 
incubadora de empresas da Universidade de São Paulo, afirma que as "Pequenas empresas são o sustentáculo de uma economia em qualquer lugar do mundo. São elas que agregam valor a produtos e serviços" (PORTAL BRASIL, 2010). Consoante a isso, no Brasil, as PMEs representam 20\% do PIB, são responsáveis por 60\% dos 94 milhões de empregos no território nacional e constituem 99\% dos seis milhões de estabelecimentos formais existentes no país (IBGE, 2010).

Mesmo diante da importância e das potencialidades das PMEs, o ambiente econômico turbulento no qual estão inseridas dificulta o seu crescimento e a sua sobrevivência ao longo do tempo. Dados demonstram que praticamente metade das empresas criadas em 1997 fechou suas portas até 2005 (MELO, 2007). Ou seja, poucas empresas brasileiras ultrapassam a sua fase de implantação, sendo vencidas pelo que se pode chamar de desafios da sobrevivência, dentre os quais, a falta de capital de giro, os impostos elevados e a falta de uma carteira mínima de clientes (SEBRAE, 2004).

Ao pequeno número de empresas sobreviventes, o inquestionável é que elas precisam necessariamente passar para o estágio seguinte, qual o seja, aquele de desenvolver uma estratégia de competitividade, para continuar crescendo qualitativa e quantitativamente, vale dizer, para vencer novos desafios, agora os desafios do crescimento (NORTH; SILVA NETO; CALLE, 2013).

A capacidade de inovação é importante para as empresas que querem crescer e se manter sustentáveis no mercado. Muller e Zenker (2001), ressaltam alguns dos fatores limitadores para a inovação; essas PMEs são aquelas relacionadas aos fluxos de informação e conhecimento - escassez de capital, ausência de qualificação em gestão e dificuldades em obter informações técnicas e know-how para inovação.

Dessa forma, várias estratégias têm sido propostas com o intuito de atacar essa problemática, por exemplo: a incubação de PMEs (TSAI et al, 2009), políticas públicas orientadas a PMEs (RUSSO; ROSSI, 2009; NICOLESCU; NICOLESCU, 2010), transferência de tecnologia com universidades e centros de pesquisa (OLAZARAN; ALBIZU; OTERO, 2009) e criação de redes de cooperação entre empresas (ZENG; XIE; TAM, 2010).

\section{PROCEDIMENTOS METODOLÓGICOS}

O presente artigo está inserido no contexto do macroprojeto "Sustainable competitiveness of SMEs in turbulent economic and social environments - a network approach" ou simplesmente "Dynamic SME", cujo objetivo é desenvolver um processo de co-evolução em direção a um modelo de gestão bem-sucedida para as PMEs, que combine a inovação europeia e seu modelo de aprendizagem organizacional com as estratégias de sobrevivência das empresas da América Latina em economias turbulentas, com base na aprendizagem em redes e clusters.

Em paralelo, este artigo também se desenvolveu junto às atividades do projeto de extensão A Economia do Conhecimento e as Empresas de Pequeno Porte e do projeto de pesquisa da Capacidade de Inovação e Crescimento das Empresas Maranhenses de Pequeno Porte, vinculados à Universidade Federal do Maranhão, e cujos objetivos são capacitar os gestores para transformar suas empresas em organizações do conhecimento e desenvolver uma metodologia que permita diagnosticar e avaliar a capacidade de inovação e de crescimento das PMEs, pela identificação das práticas da gestão do conhecimento.

Como principais contribuições para este artigo, os projetos proporcionaram um aporte práticoteórico que auxiliaram na condução sistematizada em estudo em foco. Portanto, trata-se de uma pesquisa exploratória e descritiva, de cunho qualitativo, desenvolvida em quatro etapas: (i) revisão de literatura; (ii) reestruturação do método Aprender a Crescer; (iii) consulta a especialistas; e (iv) apresentação da metodologia proposta.

A revisão de literatura foi desenvolvida com o objetivo de compreender os temas de estudo, sua relevância e a maneira como têm sido estudados no meio acadêmico- científico. Para isso, as principais fontes de informações foram os artigos publicados em bases de dados indexadas, como a ISI Web of Science, Scopue e outras.

No decorrer da revisão de literatura, identificou-se a metodologia Aprender a Crescer (HARDWIG; BERGSTERMANN; NORTH, 2011) como método de diagnóstico da capacidade de inovação e crescimento das PMEs. Esse método é constituído por dezesseis perguntas, que foram analisadas e reescritas, levando em consideração a linguagem cotidiana do pequeno empresário.

Ainda na revisão de literatura, foram identificadas práticas da gestão do conhecimento que poderiam suportar as perguntas reescritas com base no método Aprender a Crescer. Assim sendo, foi proposto pela associação dessas práticas com as perguntas reescritas, levando em consideração o quadrante com que cada pergunta estava relacionada e quais objetivos precisavam ser alcançados. 
Ademais, foram consultados gestores e empresários de algumas PMEs de diferentes setores das cidades de Imperatriz - MA e Açailândia - MA, com o objetivo de extrair possíveis práticas das suas empresas que pudessem complementar as demandas dos quadrantes do método Aprender a Crescer. Para tanto, os gestores e empresários receberam explicações sobre o método e foram convidados a indicar quais práticas são utilizadas por suas empresas para alcançar os objetivos perguntados. Essas práticas foram analisadas e implementadas na metodologia a ser apresentada.

Sugere-se, portanto, uma metodologia de diagnóstico e avaliação da capacidade de inovação e crescimento da PMEs que, de maneira complementar, combina a metodologia Aprender a Crescer com as práticas da gestão do conhecimento, levando em consideração as necessidades do pequeno empresário, tendo em vista uma futura aplicação nas PMEs.

\section{APRESENTAÇÃO E ANÁLISE DOS DADOS}

A seguir, apresenta-se a metodologia desenvolvida com a finalidade de avaliar a capacidade de inovação e de crescimento das PMEs. A apresentação está organizada fundamentalmente em função dos quatro desafios propostos pelo método Aprender a Crescer (HARDWIG; BERGSTERMANN; NORTH, 2011): (i) reconhecer oportunidades para o crescimento; (ii) desenvolver/comunicar estratégias de crescimento; (iii) realizar as oportunidades de crescimento; e (iv) gerenciar o crescimento, alocando as práticas da gestão do conhecimento que suportam e potencializam o alcance de cada desafio.

\subsection{Reconhecer oportunidades para o crescimento}

Segundo a metodologia Aprender a Crescer, esse é o primeiro desafio que as PMEs enfrentam ao crescer e inovar. 0 reconhecimento de novas oportunidades é tarefa importante e necessária que as organizações precisam enfrentar, para se manterem competitivas. Esse desafio encontra-se dividido em dois grupos: (i) reconhecer oportunidades no ambiente de negócios; e (ii) criação de novas oportunidades.

Cada um desses grupos apresenta ações que potencializam a superação do desafio de reconhecer as oportunidades para o crescimento. Com base na revisão de literatura, foram elencadas algumas das práticas da gestão do conhecimento que suportam cada uma dessas ações, apontando caminhos para as PMEs superarem esse desafio ao crescimento, como pode ser visualizado na Figura 2.

\begin{tabular}{|c|c|}
\hline \multicolumn{2}{|c|}{ RECONHECER OPORTUNIDADES PARA O CRESCIMENTO } \\
\hline $\begin{array}{l}\text { RECONHECER OPORTUNIDADES NO } \\
\text { AMBIENTE DE NEGÓCIO }\end{array}$ & CRIAÇÃO DE NOVAS OPORTUNIDADES \\
\hline $\begin{array}{l}\text { 1. Nossa empresa reconhece, de forma organizada, } \\
\text { as ameaças do nosso seguimento de mercado, além } \\
\text { de estar atenta para o que nele está sendo } \\
\text { desenvolvido. } \\
\text { a. Visitamos feiras e congressos. } \\
\text { b. Buscamos as melhores referências. } \\
\text { c. Participamos de confraternizações com empresas } \\
\text { e/ou fornecedores e/ou clientes. } \\
\text { 2. Nossa empresa adquire, de forma organizada, as } \\
\text { ideias que fornecedores, parceiros e clientes podem } \\
\text { oferecer. } \\
\text { a. Trocamos e-mails. } \\
\text { b. Elaboramos relatórios. } \\
\text { 3. Nossa empresa incentiva os funcionários a dar } \\
\text { ideias e sugestões. } \\
\text { a. Promovemos reuniões com os nossos } \\
\text { funcionários. } \\
\text { b. Matemos espaços eletrônicos. } \\
\text { c. Temos um sistema de sugestão de melhorias. }\end{array}$ & $\begin{array}{l}\text { 4. Nossa empresa ajusta suas atividades para as } \\
\text { demandas dos clientes e é capaz de captar suas } \\
\text { necessidades futuras e delas criar novas } \\
\text { oportunidades para a empresa. } \\
\text { a. Mantemos espaços eletrônicos. } \\
\text { b. Visitamos e nos socializamos com clientes. } \\
\text { c. Visitamos feiras e congressos. } \\
5 \text {. Nossa empresa se relaciona com parceiros } \\
\text { (Instituições de pesquisa, universidades, etc.) e } \\
\text { participa de redes de empresas e entidades de classes } \\
\text { (cooperativas, associações, federação da indústria, } \\
\text { etc.) para poder reconhecer oportunidades para } \\
\text { nosso crescimento e inovação. } \\
\text { a. Nossos gerentes participam de reuniões e } \\
\text { entidades de classes. } \\
\text { b. Buscamos parcerias com fornecedores. } \\
\text { c. Fazemos parcerias com instituições de apoio, } \\
\text { como o SEBRAE e universidades. }\end{array}$ \\
\hline
\end{tabular}


Dessa forma, três ações foram apresentadas no grupo para reconhecer oportunidades no ambiente dos negócios: (1) nossa empresa reconhece, de forma organizada, as ameaças do nosso segmento de mercado; (2) nossa empresa adquire, de forma organizada, as ideias que fornecedores, parceiros e clientes podem oferecer; e (3) nossa empresa incentiva os funcionários a dar ideias e sugestões.

Como forma de efetivar a ação número 1 (nossa empresa reconhece, de forma organizada, as ameaças do nosso segmento de mercado), três práticas da gestão do conhecimento foram sugeridas. A prática de visitar feiras e congressos (BALESTRIN; VERSCHOORE, 2008; BALESTRIN;VARGAS,2008), que permite aos empresários conhecer outras experiências e refletir conjuntamente sobre oportunidades e desafios.

Outra prática da gestão do conhecimento é a busca das melhores referências, como uma maneira de comparar os processos e serviços da organização e permitir identificar as melhores práticas (FUJIMOTO, 1999; BATISTA, 2006; BHIRUD; RODRIGUES;DESAI, 2005; SALIM, 2002; BALESTRIN; VERSCHOORE, 2008; GOUSSEVSKAIA, 2007; OLIVEIRA JR. et al, 2007; SKYRME; AMIDON,1997; KEYES, 2006; MAIER, 2007; OECD, 2003). Por sua vez, as confraternizações (AHMADJIAN, 2008; BALESTRIN; VERSCHOORE, 2008) permitem solidificar as relações de confiança e propiciam conversas informais a respeito do mercado.

Quanto à ação número 2 (nossa empresa adquire, de forma organizada, as ideias que fornecedores, parceiros e clientes podem oferecer), a prática da troca de e-mails é uma maneira de formalizar as informações e fortalecer o contato com fornecedores, parceiros e clientes (BALESTRIN; VERSCHOORE, 2008; BATISTA, 2006; SALIM, 2002; GOUSSEVSKAIA, 2007; SKYRME; AMIDON, 1997; OECD, 2003; DAVENPORT; PRUSAK, 1998; ANANTATMULA, 2004). A elaboração de relatórios, nesse caso, permite levar as informações captadas pelos fornecedores, parceiros e clientes para facilitar as tomadas de decisões na empresa (SOUZA, 2011).

Incentivar os funcionários a dar ideias e sugestões, ação enumerada como 3, pode ser efetivada pela prática de reuniões entre os gestores da empresa e seus funcionários, tendo em vista solucionar problemas diversos e gerar ideias novais e atuais (NONAKA; TAKEUCHI, 1995; SILVA; ROZENFELD, 2003; NONAKA; TOYAMA, 2008; GOUSSEVSKAIA, 2007; POPADIUK; CHOO, 2006).

A manutenção de espaços eletrônicos, como portais, e-mails, chats, intranet e extranet (BALESTRIN; VERSCHOORE, 2008; BATISTA, 2006; SALIM, 2002; GOUSSEVSKAIA, 2007; SKYRME; AMIDON, 1997; OECD, 2003; DAVENPORT; PRUSAK, 1998; ANANTATMULA, 2004), possibilita fortalecer as relações entre os funcionários da empresa, acelerar a comunicação e o encontro de ideias. E, ainda, conforme ressalta North (2010), um sistema de sugestões de melhorias estimula os funcionários a registrar suas ideias; uma forma simples de fazer isso é o uso de uma caixa de sugestões aberta aos funcionários e em um local de fácil acesso.

Essas práticas da gestão do conhecimento apresentadas e associadas a essas três ações são apontadas neste estudo como uma das formas de permitir a empresa reconhecer oportunidades no ambiente dos negócios.

Ainda, conforme a Figura 2, o grupo de criação de novas oportunidades apresenta as seguintes ações (4) nossa empresa ajusta suas atividades para as demandas dos clientes e é capaz de captar suas necessidades futuras e delas criar novas oportunidades para a empresa; e (5) nossa empresa relaciona-se com parceiros e participa de redes de empresas e entidades de classes para poder reconhecer oportunidades para nosso crescimento e inovação.

Uma das práticas da gestão do conhecimento que potencializa a ação número 4 pode ser a manutenção de espaços eletrônicos (BALESTRIN; VERSCHOORE, 2008; BATISTA, 2006; SALIM, 2002; GOUSSEVSKAIA, 2007; SKYRME; AMIDON, 1997; OECD, 2003; DAVENPORT; PRUSAK, 1998; ANANTATMULA, 2004), como um modo de fortalecer a relação entre a empresa e seus clientes, e agilizar o contato entre eles.

Assim, também, como oportunidade de adquirir novas informações e conhecimentos, e propiciar uma interação, é sugerida que a empresa realize visitas contínuas aos seus clientes (NONAKA; TOYAMA, 2008; BHIRUD; RODRIGUES; DESAI, 2005; OLIVEIRA JR. et al, 2007; NONAKA; TAKEUCHI, 1995; FUJIMOTO, 1999; BALESTRIN; VERSCHOORE, 2008; KON, 2007; OECD, 2003; BALESTRIN; VARGAS; FAYARD, 2008).

Por sua vez, as visitas a feiras e congressos (BALESTRIN; VERSCHOORE, 2008; BALESTRIN; VARGAS; FAYARD, 2008) proporcionam a atualização das tendências do mercado, com o objetivo de ajustar as atividades da empresa para as demandas dos clientes.

Em se tratando da ação número 5, apresentada na Figura 2, sugere-se a participação dos gerentes da empresa em reuniões de entidades de classes e a construção de parcerias com fornecedores, buscando auxiliar na melhoria dos serviços e na promoção de eventos. Já a parceria com instituições de apoio, como o Serviço de Apoio às Micro e Pequenas Empresas (SEBRAE) e universidades, também é incentivada por manter o empresário atento às oportunidades do mercado de crescimento e inovação. 


\subsection{Desenvolver e comunicar estratégias de crescimento}

Este desafio enfrentado pelas PMEs refere-se à necessidade de desenvolver estratégias e objetivos de crescimento e motivar os funcionários para o crescimento, conforme ilustrado na Figura 3. Sugere-se que as ações que auxiliam às empresas a superarem esse desafio são sustentadas por um conjunto de práticas da gestão do conhecimento.

O desafio apresenta dois grupos: (i) Desenvolver estratégias e objetivos de crescimento. (ii) Motivar os funcionários para o crescimento. Para o primeiro grupo, duas ações foram apresentadas: (6) de forma periódica nossa empresa traça estratégias e objetivos de crescimento, definindo metas e cronogramas; e (7) estamos aptos a desenvolver novos modelos de negócio que criam valor no longo prazo, como uma das formas de garantir nossa permanência no mercado.

Figura 3 - Desenvolver e comunicar estratégias de crescimento

\begin{tabular}{|c|c|}
\hline \multicolumn{2}{|c|}{ DESENVOLVER E COMUNICAR ESTRATÉGIAS DE CRESCIMENTO } \\
\hline $\begin{array}{l}\text { DESENVOLVER ESTRATÉGIAS E OBJETIVOS } \\
\text { DE CRESCIMENTO }\end{array}$ & $\begin{array}{l}\text { MOTIVAR OS FUNCIONÁRIOS PARA } \\
\text { O CRESCIMENTO }\end{array}$ \\
\hline $\begin{array}{l}\text { 6. De forma periódica (mensal, semestral, anual) } \\
\text { nossa empresa traça estratégias e objetivos de } \\
\text { crescimento, definindo metas e cronogramas. } \\
\text { a. Nossos funcionários, fornecedores e clientes } \\
\text { contribuem na realização das estratégias. } \\
\text { b. Documentamos e armazenamos informações. } \\
\text { c. Seguimos regularmente os cronogramas. } \\
\text { 7. Estamos aptos a desenvolver novos modelos de } \\
\text { negócio que criam valor a longo prazo, como uma } \\
\text { das formas de garantir nossa permanência no } \\
\text { mercado. } \\
\text { a. Buscamos as melhores referências. } \\
\text { b. Treinamos e contribuímos com a educação de } \\
\text { nossos funcionários. } \\
\text { c. Visitamos feiras e congressos. }\end{array}$ & $\begin{array}{l}\text { 8. Nossa empresa sabe motivar os funcionários para } \\
\text { atingir os objetivos da empresa. } \\
\text { a. Temos um sistema de remuneração por } \\
\text { rendimento. } \\
\text { b. Apresentamos aos nossos funcionários feefdback } \\
\text { sobre seus rendimentos. } \\
\text { c. Damos oportunidades de promoção. }\end{array}$ \\
\hline
\end{tabular}

Fontes: Os autores (2013)

A ação enumerada como 6 pode ser potencializada com a colaboração dos funcionários, fornecedores e clientes na realização das estratégias, objetivos e cronogramas da empresa (BALESTRIN; VERSCHOORE, 2008), assim como, administrar as metas e cronogramas com a contribuição dos colaboradores que proporciona à empresa uma visão mais ampla do mercado.

Documentar e armazenar informações de fontes internas e externas, formais e informais (BATISTA, 2006) é um apoio ao processo de tomada de decisões. Documentar e armazenar essas informações é uma forma de manter um acesso rápido e fácil ao conhecimento da empresa. Seguir regularmente os cronogramas foi apontado pelos gestores e empresários consultados como uma maneira de potencializar o alcance dos objetivos traçados.

A ação número 7 pode ser sustentada pela busca das melhores referências no mercado para comparar com os processos e serviços da empresa (FUJIMOTO, 1999; BATISTA, 2006; BHIRUD; RODRIGUES; DESAI, 2005; SALIM, 2002; BALESTRIN; VERSCHOORE, 2008; GOUSSEVSKAIA, 2007; OLIVEIRA JR. et al, 2007; SKYRME; AMIDON,1997; KEYES,2006; MAIER, 2007; OECD, 2003).

Treinar os funcionários e contribuir com sua educação (BATISTA, 2006; SALIM, 2002; OECD, 2003), por intermádio de parcerias com escolas e universidades, ou do reembolso das taxas de matrícula ou mensalidades em cursos relacionados com o trabalho desenvolvido na empresa, promovem a atualização contínua dos funcionários, o que poderá refletir de forma positiva nos novos modelos de negócio desenvolvidos pela empresa.

Visitar feiras e congressos (BALESTRIN; VERSCHOORE, 2008; BALESTRIN; VARGAS; FAYARD, 2008) igualmente pode proporcionar ao empresário conhecer experiências e oportunidades que auxiliam no 
desenvolvimento de novos modelos de negócio que criam valor em longo prazo, gerando fontes adicionais de vantagem competitiva à organização.

Desse modo, desenvolver estratégias e objetivos de crescimento permite enfrentar às provocações propostas pelo desafio desenvolver e comunicar estratégias de crescimento. Traçar objetivos e desafios de forma periódica e desenvolver novos modelos de negócio que criam valor a longo prazo, , são ações que contribuem para essa potencialização. Por fim, a ampliação do uso das práticas da gestão do conhecimento torna-se um caminho para superar esses desafios.

Para responder às questões relacionadas ao segundo grupo (motivar os funcionários para o crescimento), surge a ação número 8 (nossa empresa sabe motivar os funcionários para atingir os objetivos da empresa), conforme apresentado na Figura 3. A remuneração por rendimento (NORTH, 2010) é uma forma tradicional de incentivar todo o grupo de funcionários. Por sua vez, apresentação de feefdback (NORTH, 2010), como críticas e reconhecimento, aliada às oportunidades de promoção (NORTH, 2010), são maneiras de incentivar o funcionário individualmente a atingir os objetivos da empresa.

\subsection{Realizar as oportunidades de crescimento}

O terceiro desafio está dividido em dois grupos: (i) desenvolver o espírito empreendedor dos funcionários; (ii) desenvolver conhecimentos e competências. Cada um desses grupos apresenta ações que permitem realizar as oportunidades de crescimento e, para efetivar cada uma dessas ações, foram sugeridas algumas práticas da gestão do conhecimento, como pode ser visualizado na Figura 4.

Assim, o grupo relacionado ao desenvolvimento do espírito empreendedor dos funcionários conta com as seguintes ações: (9) nossos funcionários são estimulados a pensar e agir com espírito empreendedor e assumir responsabilidades; e (10) nossos gerentes sabem delegar tarefas e responsabilidades aos funcionários para que as atividades não sejam centralizadas.

Para efetivar a ação 9 apresentada, alguns gestores e empresários consultados sugeriram a política de participação nos lucros ou resultados, como forma de estimular os funcionários a pensar como empreendedores. Estimular os funcionários a continuarem sua formação (BATISTA, 2006; SALIM, 2002; OECD, 2003), por sua vez, possibilita que eles aumentem seus conhecimentos e utilizem-nos dentro da empresa. A política de premiações para ideias inovadoras também foi apontada pelos gestores e empresários consultados, como uma prática reconhecida pelos funcionários e que reflete no seu comprometimento.

Figura 4 - Realizar oportunidades de crescimento

\begin{tabular}{|c|c|}
\hline \multicolumn{2}{|c|}{ REALIZAR OPORTUNIDADES DE CRESCIMENTO } \\
\hline $\begin{array}{l}\text { DESNVOLVER O ESPIRITO EMPREENDEDOR } \\
\text { DOS FUNCIONÁRIOS }\end{array}$ & $\begin{array}{l}\text { DESENVOLVER CONHECIMENTOS E } \\
\text { COMPETÊNCIAS }\end{array}$ \\
\hline $\begin{array}{l}\text { 9. Nossos funcionários são estimulados a pensar e } \\
\text { agir com espirito empreendedor e a assumirem } \\
\text { responsabilidade. } \\
\text { a. Temos uma politica de participação nos lucros } \\
\text { ou resultados. } \\
\text { b. Estimulamos nossos funcionários a } \\
\text { continuarem sua educação. } \\
\text { c. Premiamos ideias inovadoras. } \\
\text { 10. Nossos gerentes sabem delegar tarefas e } \\
\text { responsabilidades aos funcionários para que as } \\
\text { atividades não sejam centralizadas. } \\
\text { a. Mantemos arquivos com informaçø̃es } \\
\text { curriculares dos nossos funcionários. } \\
\text { b. Realizamos reuniões periódicas. } \\
\text { c. Promovemos trocas de funcionários em } \\
\text { diversos níveis. }\end{array}$ & $\begin{array}{l}\text { 11. De forma organizada e constate, nossa empresa } \\
\text { desenvolve as competências de seus funcionários } \\
\text { para garantir o crescimento. } \\
\text { a. Usamos um sistema mestre-aprendiz. } \\
\text { b. Realizamos treinamentos, cursos workshops } \\
\text { como nossos funcionários. } \\
\text { c. Matemos parcerias com escolas e universidades. } \\
\text { 12. Nossa empresa estimula que os conhecimentos } \\
\text { sejam constantemente compartilhados entre os } \\
\text { departamentos, funções e hierarquias. } \\
\text { a. Realizamos reuniões periódicas. } \\
\text { b. Explicamos e disponibilizamos nossos } \\
\text { procedimentos. } \\
\text { c. Registramos o conhecimento da empresa. }\end{array}$ \\
\hline
\end{tabular}

Fonte: Elaboração dos autores (2013) 
No caso da ação 10, a manutenção de arquivos com informações curriculares dos funcionários (BATISTA, 2006; SALIM, 2002), como habilidades técnicas, científicas, artísticas e culturais, auxilia na delegação de responsabilidades por ser um meio fácil e rápido de acesso às competências dos funcionários; uma forma simples é uma lista on-line do pessoal.

A prática de reuniões periódicas (NONAKA; TAKEUCHI, 1995; SILVA; ROZENFELD, 2003; NONAKA; TOYAMA, 2008; GOUSSEVSKAIA, 2007; POPADIUK; CHOO, 2006) entre os gestores e alguns funcionários, para o repasse de responsabilidades, estimula a descentralização de tarefas. Já a troca de funcionários em diversos níveis (AHMADJIAN, 2008; GOUSSEVSKAIA, 2007; OECD, 2003) ajuda a disseminar novos processos de trabalho e o entendimento por parte dos funcionários da empresa intergralmente. Dessa forma, as práticas da gestão do conhecimento apresentadas possibilitam que a empresa desenvolva o espírito empreendedor dos funcionários, ao estimular responsabilidades e descentralizar suas atividades.

A Figura 4 também apresenta aspectos relacionados ao desenvolvimento de conhecimentos e competências, por meio das ações sugeridas: (11) de forma organizada e constante, nossa empresa desenvolve as competências de seus funcionários para garantir crescimento; e (12) nossa empresa estimula que os conhecimentos sejam constantemente compartilhados entre os departamentos, funções e hierarquias.

Para satisfazer a ação (11), apresentada na Figura 4, propõem-se a relação mestre-aprendiz, ação desempenhada por um profissional expert, espécie de mentor, que indica linhas de atuação, facilita e estimula o desenvolvimento do indivíduo ou do grupo (NONAKA; TAKEUCHI, 1995; BATISTA, 2006; SALIM, 2002; BHIRUD; RODRIGUES; DESAI, 2005; SILVA; ROZENFELD, 2003; GOUSSEVSKAIA 2007; OECD, 2003). Essa prática permite que o trabalho seja aprendido por observação, manuseio, imitação e prática.

Assim, também, a capacitação dos funcionários, por meio de treinamentos, cursos e workshops é uma forma de compartilhar experiências e acompanhar a qualificação deles (SILVA; ROZENFELD, 2003; NONAKA; TOYAMA, 2008; BALESTRIN; VERSCHOORE, 2008; GOUSSEVSKAIA, 2007; POPADIUK; CHO0, 2006; SALIM, 2002; AHMADJIAN, 2008; OECD, 2003; BALESTRIN; VARGAS; FAYARD, 2008). Por intermédio dessa prática, é possível aperfeiçoar e desenvolver continuamente as competências dos colaboradores.

As parcerias com escolas e universidades possibilita à empresa proporcionar a continuação da formação de seus funcionários (BATISTA, 2006; SALIM, 2002; OECD, 2003), promovendo a atualização contínua dos funcionários, o que poderá refletir no crescimento da empresa.

Em se tratando de estimular o compartilhamento dos conhecimentos entre os departamentos, funções e hierarquias, a ação (12), apresentada na Figura 4, surge com a prática das reuniões (NONAKA; TAKEUCHI, 1995; SILVA; ROZENFELD, 2003; NONAKA; TOYAMA, 2008; GOUSSEVSKAIA, 2007; POPADIUK; CHO0, 2006) entre os departamentos, hierarquias e funcionários, como um meio de contribuir para o compartilhamento do conhecimento entre os diversos setores da empresa.

Contudo, explicitar e disponibilizar os procedimentos de execução facilitam a compreensão do conhecimento da empresa por um número maior de funcionários (BATISTA, 2006; SALIM, 2002; OLIVEIRA JR. et al, 2007; KEYES, 2006; MAIER, 2007; OECD, 2003). Essa prática pode ocorrer mediante bancos de dados, manuais ou diretrizes.

Assim sendo, igualmente, os registros do conhecimento da empresa (BATISTA, 2006; SALIM 2002; KEYES, 2006; MAIER, 2007; OECD 2003; DAVENPORT; PRUSAK; 1998), por relatos de experiências sobre processos, serviços e relacionamentos com colaboradores, estimulam o compartilhamento de informações e podem servir como apoio aos novos funcionários.

\subsection{Gerenciar o Conhecimento}

O item gerenciar conhecimento diz respeito ao quarto desafio do crescimento e contém os grupos: (i) organizar o crescimento; e (ii) assegurar recursos. Cada grupo destaca duas ações voltadas para o gerenciamento do conhecimento, que podem ser sustentadas por práticas da gestão do conhecimento eleitas com base na revisão da literatura imposta por este trabalho e que se apresenta na Figura 5. 


\begin{tabular}{|c|c|}
\hline \multicolumn{2}{|c|}{ GERENCIAR O CONHECIMENTO } \\
\hline ORGANIZAR O CONHECIMENTO & ASSEGURAR RECURSOS \\
\hline $\begin{array}{l}\text { 13. Nossa empresa é capaz de adaptar seus } \\
\text { processos para os desafios da empresa. } \\
\text { a. Mantemos uma base de dados on-line. } \\
\text { b. Gerenciamos nossos documentos. } \\
\text { c. Gerenciamos nossos documentos de forma } \\
\text { eletrônicas. } \\
\text { 14. Gestores e funcionários sabem conviver com } \\
\text { o crescimento. } \\
\text { a. Buscamos as melhores referências. } \\
\text { b. Consultamos um conselheiro de campo. } \\
\text { c. Buscamos recursos financeiros externos. }\end{array}$ & $\begin{array}{l}\text { 15. Nossa empresa disponibiliza de funcionários } \\
\text { para o crescimento. } \\
\text { a. Recrutamos mão de obra. } \\
\text { b. Dispomos de uma forma de saber quando } \\
\text { precisamos de mais funcionários. } \\
\text { 16. Nossa empresa assegura o financiamento do } \\
\text { crescimento. } \\
\text { a. Buscamos fontes de financiamento. } \\
\text { b. Elaboramos um levantamento para decidir qual } \\
\text { área receberá financiamento. }\end{array}$ \\
\hline
\end{tabular}

Fonte: Elaboração dos autores (2013)

As ações para organização do crescimento foram definidas como: (13) nossa empresa é capaz de adaptar seus processos para os desafios da própria empresa; e (14) gestores e funcionários sabem conviver com o crescimento. Então, como resposta à ação 13, três práticas da gestão do conhecimento foram apresentadas. Manter uma base de dados on-line (BATISTA 2006; KON, 2007; AMATO NETO, 2007) permite à empresa ter o controle de documentos, requisições de pagamentos e estatísticas de desempenho de funcionários para auxiliar no redesenho dos seus processos.

O gerenciamento dos documentos (BATISTA, 2006; KEYES, 2006; MAIER, 2007) reduz custos e facilita o acesso a documentos importantes e na adaptação dos processos da empresa. Gerenciar esses documentos de forma eletrônica (BATISTA, 2006; SALIM 2002), por sua vez, implica adotar aplicativos de controle de emissão, edição e acompanhamento da tramitação, da distribuição, do arquivamento e do descarte de documentos.

A ação número 14 pode ser potencializada pela busca das melhores referências (FUJIMOTO, 1999; BATISTA, 2006; BHIRUD; RODRIGUES; DESAI, 2005; SALIM, 2002; BALESTRIN; VERSCHOORE, 2008; GOUSSEVSKAIA, 2007; OLIVEIRA JR. et al, 2007; SKYRME; AMIDON, 1997; KEYES, 2006; MAIER, 2007; OECD, 2003) a fim de continuamente comparar os processos e serviços da empresa e buscar as melhores práticas.

Essas ações e práticas da gestão do conhecimento corroboram para a organização do crescimento. Com a finalidade de assegurar os recursos, segundo grupo desse desafio, duas ações foram sugeridas: (15) nossa empresa disponibiliza de funcionários necessários para o crescimento; e (16) nossa empresa assegura o financiamento do crescimento.

Para assegurar o número de funcionários necessários ao crescimento, a empresa pode recrutar mão de obra mediante contratação, indicação interna e feiras em universidades (MENEZES et al., 2012) e manter uma forma de controle capaz de sinalizar a necessidade de contratar mais mão de obra (SOUZA, 2011).

Assegurar o financiamento do crescimento, ação número 16, apresentada na Figura 5, envolve as práticas referentes à busca constante de fontes atrativas de financiamento para obtenção de recursos externos e a realização de levantamentos para decisão de quais áreas devem receber recursos financeiros (MENEZES et al, 2012).

A consulta a um conselheiro de campo (NONAKA; TOYAMA 2008; BATISTA, 2006; SALIM, 2002; BHIRUD; RODRIGUES; DESAI, 2005; KEYES, 2006), espécie de mentor que aconselha nas tomadas de decisão, igualmente auxilia no processo de tomada de decisão e no crescimento da empresa. A obtenção de recursos financeiros externos (MENEZES et al, 2012), por seu turno, é uma maneira de financiar os projetos da empresa.

Apresentados os desafios, seus respectivos grupos, ações e práticas da gestão do conhecimento de forma agrupada, a metodologia desenvolvida neste trabalho tem sua forma final apresentada na Figura 6, cujo objetivo principal é possibilitar avaliar o desempenho e a relevância das práticas relacionadas à 
capacidade de reconhecer oportunidades para o crescimento.

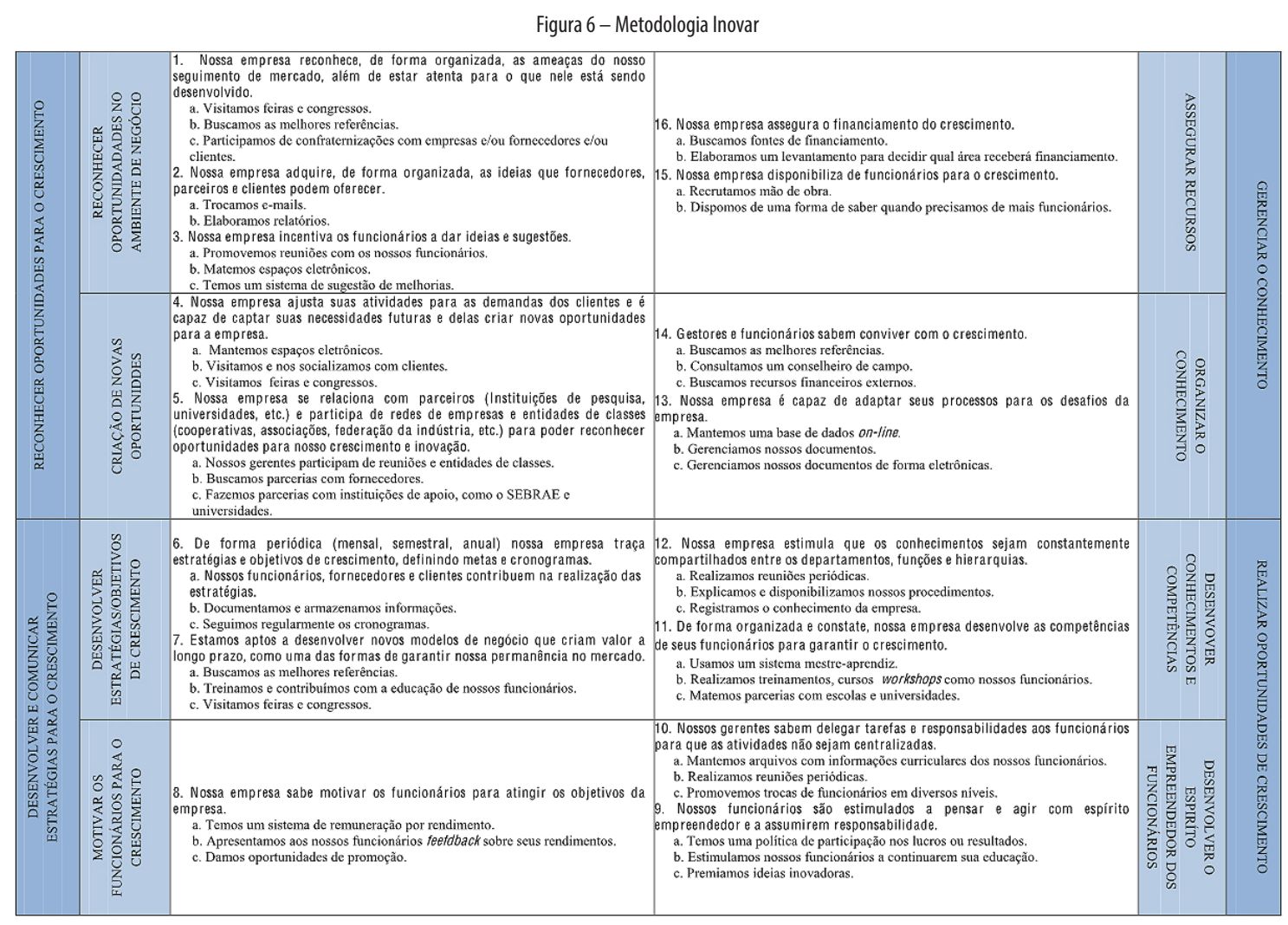

Fonte: Elaboração dos autores (2013)

\section{CONCLUSÃO}

O presente trabalho teve como objetivo o desenvolvimento de uma metodologia de diagnóstico e avaliação da capacidade de crescimento e inovação das PMEs, que combine a metodologia Aprender a Crescer e as práticas da gestão do conhecimento, levando em consideração as necessidades do pequeno empresário brasileiro. Nesse sentido, a principal contribuição da pesquisa está na apresentação de uma metodologia que possibilita as PMEs avaliar sua capacidade de reconhecer oportunidades para o crescimento, desenvolver e comunicar as estratégias e os objetivos, realizar as oportunidades e gerenciar o crescimento.

Portanto, os resultados obtidos contribuem para avaliar quais as práticas da gestão do conhecimento potencializam a capacidade de inovação e crescimento das PMEs, configurando-se em uma oportunidade para a melhoria dos serviços prestados, além da redução dos riscos de mortalidade inerentes a tais empresas.

Em paralelo, este texto corrobora com trabalhos anteriores (HARDWIG; BERGSTERMANN; NORTH, 2011; SOUZA, 2011, MENEZES et al, 2012; NORTH; SILVA NETO; CALLE, 2013), ao identificar e incorporar as práticas da gestão do conhecimento ao método Aprender a Crescer, o que permite às empresas tornaremse mais competitivas, gerenciando da melhor forma seu crescimento no mercado. 


\title{
ESTRATEGIAS DE INNOVACIÓN Y CRESCIMINETO SUSTENTADA POR PRÁCTICAS DE GESTIÓN DE CONOCIMIETO
}

\begin{abstract}
RESUMEN
Para formular o alterar su comportamiento en la intensión de adquirir mayor competitividad en el mercado, una empresa necesita trabajar una serie de requisitos. Entre ellos, se destacan la definición de objetivos y metas de la empresa, la selección de mejores estrategias a ser implementadas y la verificación de la disponibilidad de recursos necesarios. Alineada a esa realidad, una visión bastante difundida en la literatura y la idea de que los gestores de las Pequeñas y Medias Empresas - PMEs necesitan mirar más allá de los productos de la empresa y enfocar en la identificación de fuentes de conocimiento especializado que puedan atender las demandas y aprovechar ventajas competitivas con base en la mejoría del desempeño de sus procesos. Además, este artículo tiene como objetivo desenvolver una metodología de diagnostico y evaluación de la capacidad de crecimiento e innovación de las PMEs que, de manera complementar, combine la metodología Aprender a Crecer con las prácticas de la gestión del conocimiento, llevando en consideración las necesidades de los pequeños empresarios brasileños. Se trata de un estudio de carácter exploratorio y descriptivo, con abordaje cualitativo, que posibilita la comprensión de cuales prácticas de gestión de conocimiento, combinadas con la metodología Aprender a Crecer, potencializan la capacidad de innovación y crecimiento de las PMEs. Por tanto, como principal contribución de este trabajo, está la posibilidad de reconocer oportunidades para el crecimiento, desenvolver y comunicar las estrategias y los objetivos, realizar las oportunidades y gestionar el crecimiento.

Palabras claves: prácticas de gestión del conocimiento. Innovación. pequeñas y medianas empresas. ventajas competitivas.
\end{abstract}

\section{REFERÊNCIAS}

AHMADJIAN, C. L. Criação do Conhecimento Interorganizacional: conhecimento em redes. In: NONAKA, I. TAKEUCHI, H. Gestão do Conhecimento. Porto Alegre: Bookman, 2008. p. 201-216.

AMATO NETO, João. Aprendizagem Interoganizacional por meio da Colaboração Interempresarial. In: BERNARDES, Roberto (Org.). Inovação em serviços intensivos em conhecimento. São Paulo: Editora Saraiva, 2007. p. 303-324.

ANANTATMULA, V. S. P. Criteria for Measuring Knowledge Management Efforts in Organizations. 2004. Dissertation (Doctor of Science) - The School of Engineering, George Washington University, Washington.

APQC. American Productivity and Quality Center [2006]. Disponível em: <http://www.apqc.org>. Acesso em: 20 set. 2008.

BALESTRIN, A.; VARGAS, L. M.; FAYARD, P. Knowledge creation in small-firm network. Journal of Knowledge Management, Bingley, v. 12, n. 2, p. 94-106, 2008.

BALESTRIN, A.; VERSCHOORE, J. Redes de cooperação empresarial: estratégias de gestão na nova economia. Porto Alegre: Bookman, 2008.

BATISTA, Fábio Ferreira. O Desafio da Gestão do Conhecimento nas Áreas de Administração e Planejamento das Instituições Federais de Ensino Superior (IFES). (Texto para discussão, 1181). Brasília: IPEA, 2006. 
BHIRUD, S.; RODRIGUES, L.; DESAI, P. Knowledge Sharing Practices in KM: A Case Study In Indian Software Subsidiary. Journal of Knowledge Management Practice, Ontario, Dec. 2005.

BISHOP, J. et al. Ensuring the Effectiveness of a Knowledge Management Initiative. Journal of Knowledge Management, Bingley, v. 12, n. 4, p. 16-29, 2008.

DAVENPORT, T. H.; PRUSAK, L. Working knowledge: how organizations manage what they know. Boston: Harvard Business School Press, 1998.

DRUCKER, P. F. Sociedade pós-capitalista. São Paulo: Pioneira, 1994.

FELDMAN, M. S.; PENTLAND, B. T. Reconceptualizing Organizational Routines as a Source of Flexibility and Change. Administrative Science Quarterly, Published by Johnson Graduate School of Management, Cornell University, Ithaca, v. 48, n. 1, p. 94-118, 2003.

FUJIMOTO, Takahiro. The Evolution of Manufacturing System at Toyota. New York: Oxford University Press, 1999.

GOUSSEVSKAIA, Anna. Aprendizagem Interoganizacional por meio da Colaboração Interempresarial. In: BERNARDES, Roberto (Org.). Inovação em serviços intensivos em conhecimento. São Paulo: Saraiva, 2007. p. 259-281.

GRANT, R. Toward a Knowledge-Based Theory of the Firm. Strategic Management Journal, Chicago, v. 17, Winter Special Issue, p. 109-122, 1996.

HARDWIG, T.; BERGSTERMANN, M.; NORTH, K. Wachstum lernen: Eine praxiserprobte Handlungsanleitung zur Entwicklung dynamischer Fähigkeiten in KMU; mit vielen Fallbeispielen. Wiesbaden: Gabler, 2011.

IBGE. Instituto Brasileiro de Geografia e Estatística. Micro empresa. Disponível em: <http://www.ibge. gov.br/home/estatistica/economia/microempresa/default.shtm>. Acesso em: 20 jul. 2013.

KEYES, Jessica. Knowledge Management, Business Intelligence, and Content Management: the IT Practitioner's Guide. [S.l.]: Auerbach Publications, 2006.

KOGUT, Bruce; ZANDER, Udo. Knowledge of the firm, combinative capabilities, and the replication of technology. Organization Science, Pennsylvania, v. 3, n. 3, Aug. 1992.

KON, Anita. Aprendizagem Interoganizacional por meio da Colaboração Interempresarial. In: BERNARDES, Roberto (Org.). Inovação em serviços intensivos em conhecimento. São Paulo: Editora Saraiva, 2007. p. 79-115.

LLORIA, M. Begoña. A review of the main approaches to knowledge management. Knowledge Management Research \& Practice, Basingstoke, v. 6, n. 1, p. 77-89, 2008.

MAIER, R. Knowledge Management Systems Information and Communication Technologies for Knowledge Management. Inssbruck, Austria: Springer, 2007.

MELO, L. As empresas brasileiras têm vida curta. [2007]. Disponível em: <http://oglobo.globo.com/ economia/empresas-brasileiras-tem-vida-curta-4137543 >. Acesso em: 17 jan. 2013. 
MENEZES, L. et al. Aprender a Crescer: análise empírica das capacidades dinâmicas de pequenas e médias empresas. In: Reunião Anual da Rede Pymes Mercosul, 17., 2012, São Paulo. Anais da XVII Reunião Anual da Rede Pymes Mercosul. São Paulo: USP, 2012.

MULLER, E.; ZENKER, A. Business services as actors of knowledge transformation: the role of KIBS in regional and national innovation systems. Research Policy, Durham, NC, v. 30, n. 9, p. 1501-1516, Dec. 2001.

NICOLESCU, O.; NICOLESCU, C. Determinants of the National SME's Strategy. Metalurgia International, Romania, v. 15, p. 100-104, 2010.

NONAKA, I; TOYAMA, R. Criação do Conhecimento como Processo Sintetizador. In: NONAKA, I.; TAKEUCHI, H. Gestão do Conhecimento. Porto Alegre: Bookman, 2008. p. 91-117.

NONAKA, I.; TAKEUCHI, H. The Knowledge-Creating Company: How Japanese Companies Create the Dynamics of Innovation. Oxford: Oxford University Press, 1995.

NORTH, K. Gestão do conhecimento: um guia prático rumo à empresa inteligente. Rio de Janeiro: Qualitymark, 2010.

NORTH, K.; SILVA NETO, E.; CALLE, G. D. Vencendo os desafios do crescimento: o método "aprender a crescer" para pequenas e médias empresas brasileiras. Navus - Revista de Gestão e Tecnologia, Florianópolis, SC, v. 3, n. 1, p. 6-19, jan./jun. 2013.

OECD. Measuring Knowledge Management in the Business Sector: First Steps. Canada: OECD Publications Service, 2003.

OLAZARAN, M.; ALBIZU, E.; OTERO, B. Technology Transfer between Technology Centres and SMEs: Evidence from the Basque Country. European Planning Studies, Oxfordshire, UK, v. 17, n. 3, p. 345-363, 2009.

OLIVEIRA JR. et al. Aprendizagem Interoganizacional por meio da Colaboração Interempresarial. In: BERNARDES, Roberto (Org.). Inovação em serviços intensivos em conhecimento. São Paulo: Editora Saraiva, 2007. p. 283-302.

PARRILLI, M. D.; ARANGUREN, M. J.; LARREA, M. The Role of Interactive Learning to Close the 'Innovation Gap' in SME-Based Local Economies: A Furniture Cluster in the Basque Country and its Key Policy Implications. European Planning Studies, Oxfordshire, UK, v. 18, n. 3, p. 351-370, 2010.

POPADIUK, S.; CHOO, C. W. Innovation and Knowledge Creation: how are these concepts related? International Journal of Information Management, Hong Kong, v. 26, p. 302-312, 2006.

PORTAL BRASIL. Mapa das micro e pequenas empresas. [2012]. Disponível em: <http://www.brasil.gov. br/economia-e-emprego/2012/02/o-mapa-das-micro-e-pequenas-empresas >. Acesso em: 21 jul. 2012.

RUSSO, M.; ROSSI, F. Cooperation networks and innovation: A complex systems perspective to the analysis and evaluation of a regional innovation policy programme. Evaluation, Torino, v. 15, n. 1, p. 75-99, 2009.

SALIM, J. J. Era Digital: o conhecimento em ação. RAE Executivo, São Paulo, v. 1, n. 2, p. 55-59, 2002.

SANTOS, L. C.; VARVAKIS, G. Redes de pequenas e médias empresas: organizações virtuais vs. clusters. In: Encontro Nacional de Engenharia da Produção, 19., 1999, Rio de Janeiro. Anais do XIX ENEGEP. Rio de 
Janeiro: ABEPRO, 1999. p. 1-12. Disponível em: <www.abepro.org.br/biblioteca/ENEGEP1999_A0450. PDF >. Acesso em: 21 out. 2013.

SCHREIBER, G. et al. Knowledge engineering and management: the commonKADS methodology. Cambridge, MA: MIT Press, 2002.

SEBRAE. Fatores condicionantes e taxa de mortalidade de empresas no Brasil. Publicações Sebrae. Brasília: SEBRAE/NA, 2004.

SILVA, S. L.; ROZENFELD, H. Modelo de avaliação da gestão do conhecimento no processo de desenvolvimento do produto: aplicação em um estudo de caso. Revista Produção, São Paulo, v. 13 n. 2, p. 6-20, 2003.

SKYRME, David; AMIDON, Debra. The Knowledge Agenda. The Journal of Knowledge Management, v. 1, n. 1, Sept. 1997.

SOUZA, L. L. C. Mecanismos de Coordenação e Práticas da Gestão do Conhecimento na Cadeia de Valor Terceirizada: estudo no setor elétrico. 2011. Tese (Doutorado em Engenharia e Gestão do Conhecimento) - Universidade Federal de Santa Catarina, Florianópolis.

TEECE, D. J. Explicating dynamic capabilities: The nature and microfoundations of (sustainable) enterprise performance. Strategic Management Journal, West Lafayette, v. 28, n. 13, p. 1319-1350, 2007.

TEECE, D. J.; PISANO, G.; SHUEN, A. Dynamic capabilities and strategic management. Strategic Management Journal, West Lafayette, v. 18, n. 7, p. 509-533, 1997.

TSAI, F. S. et al. The co-evolution of business incubation and national innovation systems in Taiwan. Technological Forecasting and Social Change, Stony Brook, NY, v. 76, n. 5, p. 629- 643, 2009.

WANG, Y. L.; WANG, Y. D.; HORNG, R. Y. Learning and innovation in small and medium enterprises. Industrial Management \& Data Systems, Shreveport, USA, v. 110, n. 1-2, p. 175-192, 2010.

ZENG, S. X.; XIE, X. M.; TAM, C. M. Relationship between cooperation networks and innovation performance of SMEs. Technovation, Philadelphia, PA, v. 30, n. 3, p. 181-194, Mar. 2010.

Agradecimentos:

1. À professora Jaqueline Rossato, por suas contribuições e ensinamentos fundamentais.

2. Ao PIBIC/UFMA e PROEX/UFMA, pelo apoio por meio das bolsas de iniciação científica. 